\title{
MODELAGEM CINÉTICA E INFLUÊNCIA DA TEMPERATURA DE SECAGEM NO RENDIMENTO DO EXTRATO DA CASCA DE ROMÃ (Punica granatum L.)
}

\author{
T. K. G. SANTOS ${ }^{1}$ e R. N. CUNHA ${ }^{1}$ \\ ${ }^{1}$ Centro Universitário de Patos de Minas, Faculdade de Engenharia Química \\ Departamento de Engenharia Química \\ E-mail para contato: tamarakatlin@ hotmail.com
}

\begin{abstract}
RESUMO - A Punica granatum L., conhecida popularmente como romã é uma fruta que possui características medicinais no tratamento de tosses. Estudos apontam a presença de metabólitos especiais nas cascas de romã, fato esse que contribui para o seu uso em fitocosméticos e fármacos. Dentro desse contexto, o presente trabalho objetiva determinar a cinética de secagem e avaliar a influência da temperatura sobre o rendimento do extrato obtido a partir da casca de romã. Foram avaliadas as temperaturas de $50,60,70$ e $80^{\circ} \mathrm{C}$. Aos dados experimentais foram ajustados modelos matemáticos, cujas magnitudes do coeficiente de determinação $\left(\mathrm{R}^{2}\right)$ foram utilizadas para verificar o grau de ajuste dos modelos. Os resultados obtidos apontam que as cascas de romã possuem comportamento de secagem semelhante ao da maioria dos produtos agrícolas; o modelo matemático de secagem proposto por Midilli et al. e por Page foram os que melhores se ajustaram aos dados experimentais. A temperatura do ar de secagem de $60^{\circ} \mathrm{C}$ contribuiu de modo favorável sobre o rendimento do extrato fitoquímico.
\end{abstract}

\section{INTRODUÇÃO}

A romãzeira (Punica granatum L.) é um arbusto lenhoso, ramificado, da família Punicaceae, se adapta a climas tropicais, subtropicais e também em cenário árido. Suas propriedades medicinais vêm sendo estudadas desde os primórdios de sua descoberta para tratamento de doenças que vão desde a tosse até infecções mais complexas (Lorenzi e Matos, 2008).

Além da polpa da fruta, a casca, flor e folhas da romã possuem características terapêuticas utilizadas para tratar vários problemas de saúde, predominantemente gastrintestinais e também no campo odontológico. A cura de enfermidades acontece pelo fato da romã produzir diferentes metabólitos especiais, substâncias que integram o metabolismo vegetal conferindo proteção para as plantas, além de possuírem atividades biológicas que podem beneficiar também a saúde humana de maneira a maximizar seu efeito na produção de fitocosméticos e fármacos.

A triagem de tais metabólitos é feita realizando-se uma pesquisa fitoquímica com o extrato obtido da parte a ser estudada da fruta. A prospecção fitoquímica tem como objetivo conhecer os compostos químicos das espécies vegetais e avaliar sua presença nos mesmos, identificando os grupos de metabólitos especiais relevantes, úteis enquanto marcadores 
químicos no monitoramento das plantas medicinais, em processo de domesticação na qualidade da matéria prima medicinal e na prospecção da biodiversidade (Murta, 2016).

Utilizada de forma a auxiliar no processo de aproveitamento da casca da romã, Celestino (2010) afirma que a secagem é uma operação unitária que atua de forma a retirar o líquido agregado ao alimento por meio da vaporização térmica. Esta vaporização, no entanto, deve ocorrer em uma temperatura inferior àquela de ebulição do líquido na pressão do sistema. Pacheco (2010) acrescenta que a secagem apresenta como vantagens, a manutenção dos constituintes minerais bem como a inibição de atividade microbiana. Entretanto estudos mostram que a temperatura pode exercer influências sobre os rendimentos dos extratos (Borsato, 2003). O presente trabalho tem como objetivo geral (i) determinar e modelar a cinética de secagem para o reaproveitamento da casca de romã e (ii) avaliar os efeitos da temperatura sobre o rendimento do extrato.

\section{MATERIAIS E MÉTODOS}

\subsection{As cascas de romã}

O presente estudo foi desenvolvido no Laboratório de Engenharia Química (LEQ) do Centro Universitário de Patos de Minas (UNIPAM). A romã foi adquirida de um produtor local. Os frutos foram lavados em água corrente e em seguida fracionados transversalmente a fim de se retirar a polpa. Após a retirada da polpa as cascas foram dividas em quatro partes simétricas.

\subsection{A cinética de secagem}

As cascas da romã foram dispostas em monocamada em secador de bandejas (Desidratador Pardal), com circulação forçada de ar. Os ensaios experimentais de cinética de secagem foram realizados nas temperaturas de 50,60, 70 e $80^{\circ} \mathrm{C}$. Para tanto, a amostra foi pesada em intervalos de tempos regulares com auxílio de uma balança (Digimed DG-15). A massa seca foi obtida pela secagem à $105^{\circ} \mathrm{C}$ até obtenção de peso constante. A partir dos experimentos cinéticos de secagem foi possível determinar a umidade total $X_{t}$, a umidade livre $X$ e a taxa de secagem $T S$, por meio da Equação 1, Equação 2 e Equação 3, respectivamente. (Geankoplis, 1993). A razão de umidade RU foi determinada pela razão entre as umidades livres no instante $t$ no tempo inicial, conforme expresso pela Equação 4.

$$
\begin{aligned}
& X_{t}=\frac{W-W_{s}}{W_{s}} \\
& X=X_{t}-X^{8} \\
& T S=-\frac{L_{s}}{A} \frac{d X}{d t} \\
& R U=\frac{X_{t}-X^{8}}{X_{i}-X^{8}}
\end{aligned}
$$

Para predição da cinética de secagem foram avaliados os modelos matemáticos (Page, Midilli, Henderson e Pabis e Newton e Lewis), sendo o grau de ajuste dos modelos 
determinado por meio das magnitudes do coeficiente de determinação $\left(\mathrm{R}^{2}\right)$. As constantes dos modelos são intrínsecas a cada modelo, podendo ser determinadas por regressão não-linear de um gráfico de $R U$ em função de $t$ (Tabela 01).

Tabela 1- Modelos matemáticos utilizados na predição da cinética de secagem

\begin{tabular}{|c|c|}
\hline $\begin{array}{c}\text { Designação do } \\
\text { Modelo }\end{array}$ & Modelo \\
\hline \hline Page & $R U=e^{-k t^{n}}$ \\
\hline Midilli & $R U=a e^{-k t^{n}}+b t$ \\
\hline Henderson e Pabis & $R U=a e^{(-K t)}$ \\
\hline Newton e Lewis & $R U=e^{(-K t)}$ \\
\hline
\end{tabular}

$R U$ : razão de umidade. $t$ : tempo (min). $a, b\left(\min ^{-1}\right), n, k\left(\min ^{-1}\right), k_{0}\left(\min ^{-1}\right), k_{l}\left(\min ^{-1}\right)$ : constantes características. Fonte: Faria et al. (2012).

O coeficiente de difusão efetivo foi obtido por meio do ajuste do modelo matemático da difusão líquida, descrito pela Equação 5. Essa equação é a solução analítica para a segunda lei de Fick considerando-se a forma geométrica do produto como aproximação a uma placa plana (Brooker et al., 1992). Por se tratar de uma série infinita, o número finito de termos $(n=12)$ no truncamento poderá determinar a precisão dos resultados.

$$
R U=\frac{X_{t}-X^{8}}{X_{i}-X^{*}}=\frac{8}{\pi^{2}} \sum_{n=0}^{\infty} \frac{1}{(2 n+1)^{2}} \exp \left[-\frac{D \pi^{2}(2 n+1)^{2} t}{4 L_{0}^{2}}\right]
$$

O coeficiente de difusividade está estreitamente relacionado com a locomoção aleatória das moléculas, e consequentemente com a energia de ativação $E_{a}$. Para avaliar a influência da temperatura no coeficiente de difusão efetivo utilizou-se a equação de Arrhenius, descrita conforme a Equação 6.

$D=D_{0} \exp \left[-\frac{E_{\alpha}}{R T}\right]$

\subsection{Obtenção do extrato}

As amostra de cascas secas à temperatura de 50 e $60^{\circ} \mathrm{C}$ foram trituradas em moinho de facas (Willye-NL-226-02) obtendo-se farelos. Em seguida realizou-se extração exaustiva em extrator Soxhlet utilizando Clorofórmio como solvente. O excesso de solvente foi retirado ao final do processo com auxílio de Rotavapor (Fisatom 550) e os extratos obtidos foram mantidos sobre abrigo da luz e umidade. O rendimento foi calculado através da Equação 7.

$$
\% R E=\frac{g \text { de extrato seco }}{g \text { inicial total }} \times 100
$$




\section{RESULTADOS E DISCUSSÕES}

Análises de umidade da casca de romã in natura resultaram em 61,2 $\pm 4,5 \%$, compatíveis com os de Santos et al. (2010) que obteve 73,93\%. A cinética de secagem da casca de romã para as temperaturas consideradas no estudo é apresentada na forma de razão de umidade (RU) em função do tempo (Figura 1A). Infere-se que o processo de secagem se comportou de maneira uniforme e contínua para todas as temperaturas. Verifica-se diminuição no tempo gasto para secar a casca de romã, em função da elevação da temperatura, ou seja, a maior taxa de secagem (Figura 1B) é obtida na temperatura mais elevada. Observa-se, também, que o tempo gasto $(22,3 \mathrm{~h})$ para secar a casca na temperatura mais baixa $\left(50^{\circ} \mathrm{C}\right)$ é quase o triplo do tempo despendido para secá-lo a temperatura mais elevada $\left(80^{\circ} \mathrm{C}\right)$, que foi de $7,6 \mathrm{~h}$. Mediante a exposição desses dados, verifica-se que a temperatura afeta de modo expressivo o processo de secagem, cujos resultados se encontram respaldados por vários pesquisadores, que consideram a temperatura do ar de secagem como o parâmetro que exerce maior influência na cinética de secagem de alimentos (Gouveia et al.,1999)

Figura 1 - Curva de secagem para a casca da romã nas diferentes condições de processo
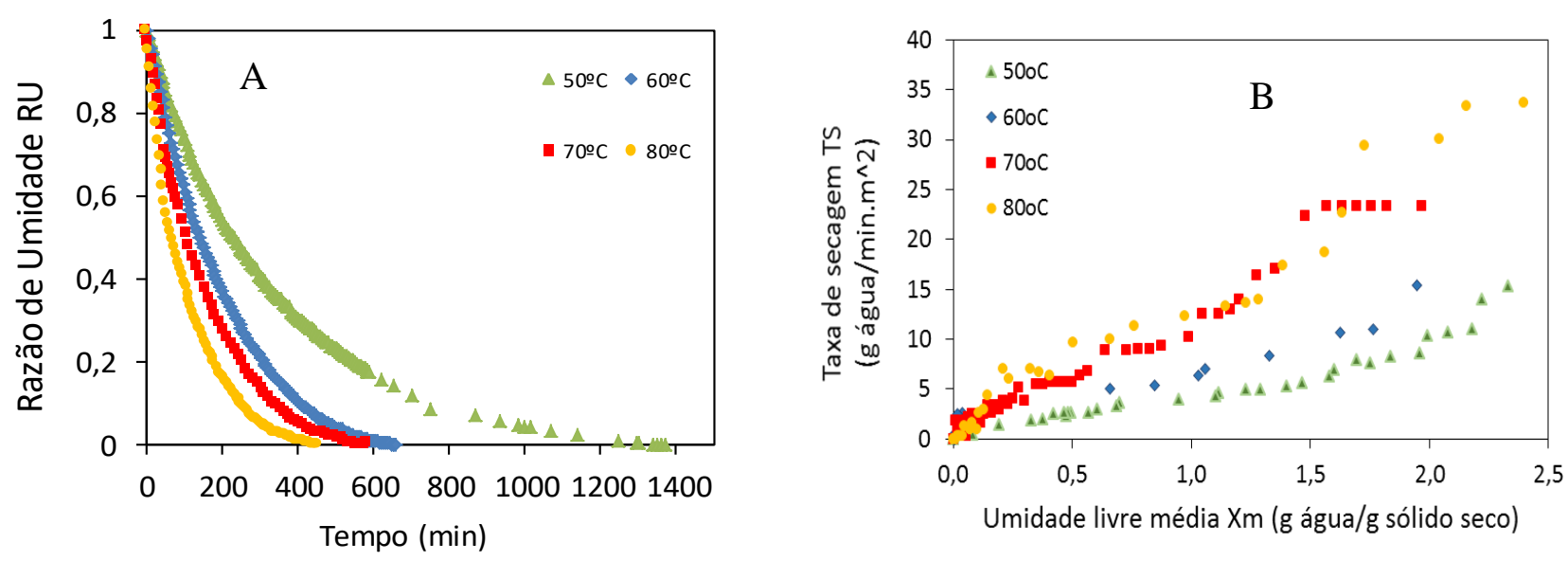

Avaliações qualitativas da cor indicam que a temperatura de $50^{\circ} \mathrm{C}$ favoreceu permanência das características físicas. Resultados semelhantes foram obtidos por Murta (2016) na secagem de casca de maracujá amarelo, em que o aumento da temperatura do ar de secagem ocasionou o escurecimento do produto. Este escurecimento deve-se, provavelmente, a exposição do resíduo por longo tempo à temperatura de secagem, favorecendo a reação de Maillard, devido à presença de açúcares e proteínas no resíduo (Ferreira e Pena, 2010).

Na Tabela 2 é apresentado o resumo do ajuste e os parâmetros estatísticos dos modelos por meio de regressão não linear aos dados experimentais da secagem de casca de romã em camada fina, considerando as diferentes temperaturas de secagem. Dentre os modelos avaliados e conforme os resultados obtidos para os coeficientes de determinação ajustado, a equação de Midilli et al. seguida pela equação de Page foram as que melhor representaram os dados experimentais para descrever o processo de secagem da casca de romã para a faixa de 
temperatura $\left(50\right.$ a $\left.80^{\circ} \mathrm{C}\right)$ estudada. A superioridade do modelo de Midilli et al. em relação ao de Page é explicada devido ao fato de a equação ter um maior número de parâmetros. Resultados semelhantes foram obtidos por Martins et al. (2013) que trabalhando a secagem de casca de mulungu verificaram que o modelo de Midilli et al. se ajustou muito bem aos dados de secagem.

Para verificar a uniformidade de variação da difusividade com a temperatura na forma de "1n Def" apresenta-se a Figura 2, descrita em função do recíproco da temperatura absoluta $\left(\mathrm{T}^{-1}\right)$. A energia de ativação calculada da reta foi de $48,9 \mathrm{~kJ} \mathrm{~mol}^{-1}\left(\mathrm{R}^{2}=0,953\right)$. Em processos de secagem quanto menor a energia de ativação maior a difusividade de água no produto. Santos e Morais (2014) estimaram a energia de ativação para casca de laranja e obtiveram $92,8 \mathrm{~kJ}$ mol-1. Segundo Zogzas et al. (1996) para produtos agrícolas o valor para a energia de ativação varia de 12,7 até $110 \mathrm{~kJ} \cdot \mathrm{mol}^{-1}$.

Tabela 2 - Parâmetros e coeficientes de determinação dos modelos ajustados.

\begin{tabular}{|c|c|c|c|c|c|}
\hline Modelo & Temperatura & $50^{\circ} \mathrm{C}$ & $60^{\circ} \mathrm{C}$ & $70^{\circ} \mathrm{C}$ & $80^{\circ} \mathrm{C}$ \\
\hline \hline \multirow{4}{*}{ Page } & $\mathrm{k}(\min -1)$ & 0,0034 & 0,0052 & 0,0027 & 0,0114 \\
\cline { 2 - 6 } & $\mathrm{n}$ & 0,9765 & 1,0448 & 1,1253 & 0,9632 \\
\cline { 2 - 6 } & $\mathrm{R}^{2}$ & 0,9994 & 0,9979 & 0,9975 & 0,9971 \\
\hline \multirow{4}{*}{ Midilli } & $\mathrm{a}$ & 1,0096 & 1,0088 & 0,9981 & 1,0126 \\
\cline { 2 - 6 } & $\mathrm{b}$ & 0 & $-0,0001$ & $-0,0001$ & $-0,0001$ \\
\cline { 2 - 6 } & $\mathrm{k}(\min -1)$ & 0,0042 & 0,0075 & 0,0039 & 0,0165 \\
\cline { 2 - 6 } & $\mathrm{n}$ & 0,9411 & 0,965 & 1,0404 & 0,8785 \\
\cline { 2 - 6 } & $\mathrm{R}^{2}$ & 0,9997 & 0,9994 & 0,9997 & 0,9992 \\
\hline \multirow{3}{*}{ Newton e Lewis } & $\mathrm{k}(\min -1)$ & 0,003 & 0,0066 & 0,0053 & 0,0095 \\
\cline { 2 - 6 } & $\mathrm{R}^{2}$ & 0,9992 & 0,9973 & 0,9928 & 0,9966 \\
\hline \multirow{3}{*}{ Henderson e Pabis } & $\mathrm{a}$ & 0,992 & 1,0135 & 1,0407 & 0,9764 \\
\cline { 2 - 6 } & $\mathrm{k}(\min -1)$ & 0,003 & 0,0067 & 0,0055 & 0,0093 \\
\cline { 2 - 6 } & $\mathrm{R}^{2}$ & 0,9993 & 0,9975 & 0,9944 & 0,9972 \\
\hline
\end{tabular}

Figura 2- Representação de Arrhenius para a relação entre a difusividade efetiva das secagens de casca de romã.

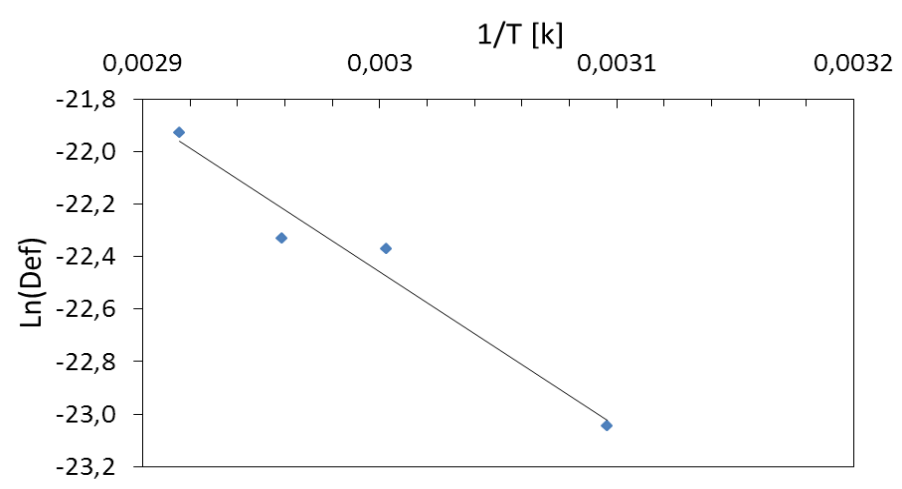

Com relação ao rendimento dos extratos, verifica-se que a temperatura não exerceu efeito significativo, pois foram obtidos os rendimentos de 1,32 e $1,59 \%$, para 50 e $60^{\circ} \mathrm{C}$, respectivamente. Entretanto, a temperatura de $60^{\circ} \mathrm{C}$ se mostra mais vantajosa do ponto de 
vista prático, pois o tempo de secagem a $60^{\circ} \mathrm{C}$ é 2 vezes menor quando confrontado com a temperatura de $50^{\circ} \mathrm{C}$.

\section{CONSIDERAÇÕES FINAIS}

Do estudo concluiu-se que: a) o comportamento das curvas de secagem das cascas de romã foi semelhante ao da maioria dos produtos agrícolas; b) o modelo matemático de secagem proposto por Midilli et al. e por Page foram os que melhores se ajustaram aos dados experimentais; c) a secagem a $60^{\circ} \mathrm{C}$ exerceu efeito favorável frente a temperatura de $50^{\circ} \mathrm{C}$, em se tratando de rendimento do extrato fitoquímico proveniente da casca de romã.

\section{REFERÊNCIAS}

BORSATO, A V. Secagem de camomila sob diferentes temperaturas e vazões específicas do ar. 2003. 77 f. Dissertação (Mestrado) - Curso de Agronomia, Departamento de Fitotecnia e Fitossanitarismo, Universidade Federal do Paraná, Curitiba, 2003.

BROOKER, D. B.; BAKKER-ARKEMA, F. W.; HALL, C. W. Drying and storage of grains and oilseeds. Westport : AVI, 1992. $450 \mathrm{p}$.

CELESTINO, S. M. C. Princípios de secagem de alimentos. 51 f. Ministério da Agricultura, Pecuária e Abastecimento, Empresa Brasileira de Pesquisas Agropecuárias (EMBRAPA), Planaltina, 2010.

FARIA, Rute Q. de et al. Cinética de secagem de sementes de crambe. Revista Brasileira de Engenharia Agrícola e Ambiental, v. 16, n. 5, p.573-583, fev. 2012.

FERREIRA, M. F. P; PENA, R. S. Estudo da secagem da casca do maracujá amarelo. Revista Brasileira de Produtos Agroindustriais, Campina Grande, v. 12, n. 1, p.15-28, nov. 2010. Anual.

GEANKOPLIS, C. J. Transport and Unit Operations; $3^{\mathrm{a}}$ Edição; Prentice-Hall, 1993.

LORENZI, H.; MATOS, F J A. Plantas medicinais no Brasil: Nativas e Exóticas. 2. ed. Nova Odessa: Plantarum, 2008. $544 \mathrm{p}$.

MARTINS, J. J. A.; MARQUES, J. I.; SANTOS, D. C.; ROCHA,A. P. T. Modelagem Matemática da secagem de cascas do mulungu. 2014. 9 f. TCC (Graduação) - Curso de Engenharia Agrícola, Universidade Federal de Campina Grande, Campina Grande, 2013.

MURTA, P. O. G. B. Influencia da temperatura no estudo fitoquímico da casca de maracujá azedo (passiflora edulis flavicarpa). 2016. 29 f. TCC (Graduação) - Curso de Engenharia Química, Centro Universitário de Patos de Minas, Patos de Minas, 2016.

PACHECO, C.R.F. Apostila de conceitos básicos de secagem, São Paulo, Departamento de Engenharia Química da Escola Politécnica da Universidade de São Paulo, 2010.

SANTOS, E. H. B.; BATISTA, F. P. R.; PEREIRA, L. M.; CAMPOS, L. M. A.; CASTRO, M. S.; AZEVÊDO, L. C. Composição físico-química dos frutos da romã. 2010. 7 f. Monografia (Especialização) - Curso de Engenharia de Alimentos, Coordenação de Tecnologia em Alimento S, Instituto Federal do Sertão, Campus Petrolina, Petrolina, 2010.

SANTOS, C. M; MORAIS, L. C de. Parâmetros termodinâmicos da casca de laranja desidratada. 2014. 1 v. Dissertação (Mestrado) - Curso de Engenharia Ambiental, Departamento de Engenharia Ambiental, Universidade Estadual Paulista "júlio de Mesquita Filho", Sorocaba, 2014.

ZOGZAS, N. P.; MAROULIS, Z. B.; MARINOS-KOURIS, D. Moisture diffusivity data compilation in foodstuffs. Drying Technology, v.14, p.2225-2253, 1996. 